\title{
Two-Axis Solar Heat Collection Tracker System for Solar Thermal Applications
}

\author{
Tsung-Chieh Cheng, Wei-Cheng Hung, and Te-Hua Fang \\ Department of Mechanical Engineering, National Kaohsiung University of Applied Sciences, Kaohsiung 807, Taiwan \\ Correspondence should be addressed to Tsung-Chieh Cheng; tcchengme@cc.kuas.edu.tw
}

Received 15 September 2013; Accepted 15 October 2013

Academic Editor: Teen-Hang Meen

Copyright ( 2013 Tsung-Chieh Cheng et al. This is an open access article distributed under the Creative Commons Attribution License, which permits unrestricted use, distribution, and reproduction in any medium, provided the original work is properly cited.

\begin{abstract}
An experimental study was performed to investigate the effect of using a continuous operation two-axes tracking on the solar heat energy collected. This heat-collection sun tracking which LDR (light dependent resistor) sensors installed on the Fersnel lens was used to control the tracking path of the sun with programming method of control with a closed loop system. The control hardware was connected to a computer through Zigbee wireless module and it also can monitor the whole tracking process information on a computer screen. An experimental study was performed to investigate the effect of using two-axes tracking on the solar heat energy collected. The results indicate that sun tracking systems are being increasingly employed to enhance the efficiency of heat collection by polar-axis tracking of the sun. Besides, the heating power was also measured by designed power measurement module at the different focal length of Fresnel lens, and the design of shadow mask of LDR sensors is an important factor for solar photothermal applications. Moreover, the results also indicated that the best time to obtain the largest solar irradiation power is during 11:00 -13:00 in Taiwan.
\end{abstract}

\section{Introduction}

Because of the energy crisis and the environmental awareness, to develop renewable energies has become one of the important targets in the future. There are many approaches that could be developed for renewable energies. Finding energy sources to satisfy the world's growing demand is one of society's foremost challenges for the next halfcentury. Solar energy is clean, renewable, and abundant in every part of our world. There are two main energy types of solar energy collection. One is photoelectric conversion and the other is photothermal conversion. For photothermal application, solar stirling engine which is a photothermal engine that converts sunlight heat energy into mechanical motion to form the electrical power by using air or an inert gas as the working fluid operating on a highly efficient thermodynamic cycle is a kind of green power. For photothermal applications, in order to receive heat energy from the sunlight, the solar tracker, a device in an optimum position perpendicular to the solar radiation during daylight hours, is necessary to increase the collected heat energy. Therefore, in this paper, an experimental study was performed to investigate the effect of using a continuous operation two-axes tracking on the solar heat energy collected.

Over the years, several researchers have studied the solar tracking systems with different modes and electromechanical module to improve the efficiency of solar systems. The design of tracker was based on some criteria: low cost, easy maintenance, modular, low energy consumption, and easy adjustment in case of different location [1]. From previous studies, there are two tracking types to track the sun. One is active type and the other is passive type [2]. In a passive system the tracker follows the sun from east to west without using any type of electric motor to power the movement, but the active type needs motor, control IC, track procedure, and detect components responding to the solar direction. Passive solar trackers are based on thermal expansion of a matter (usually Freon), on low boiling point compressed gas fluid, or on shape memory alloys. Clifford and Eastwood [3] presented the passive solar tracker design which incorporates two bimetallic strips made of aluminum and steel, positioned on a wooden frame symmetrically on either side of a central horizontal axis. The efficiency of this passive solar tracker with tracking method had the potential to increase solar panel 
efficiency by up to 23\%. Mwithiga and Kigo [4] designed and constructed a dryer which consisted of a gauge 20 mild steel flat absorber plate formed into a topless box and the results showed that the solar dryer can be used to successfully dry grains. Poulek [5] developed a single axis passive solar tracker based on a shape memory alloy (SMA). It was concluded the efficiency of the SMA actuators $(-2 \%)$ is approximately two orders of magnitude higher than that of bimetallic actuators. However, the passive tracker had many drawbacks, like low tracking efficiency than active tracker, susceptible to high winds, or sluggish in getting moving in cold temperatures. Therefore, the approach of solar tracker was trend to develop at active mode solar tracker.

Active tracking systems are powered by small electric motors and require some type of control module to direct them. The controller had two modes that can identify the active tracker to location of the sun. One is electrooptical sensors such as solar cell or LDR (Light Dependent Resistance) or photodiodes based on the structure of trackers (closed loop system); another is according to the function which adopted the azimuth and solar altitude angles or polar axis (open loop system). Abouzeid [6] presents a new tracking system with a firm step movement of either $15^{\circ}$ or $7.5^{\circ}$. The system is controlled automatically using a programmable logic array plus an EEPROM carrying all necessary commands required for different operation cases (Abouzeid, 2001). Oner et al. [7] design a spherical motor controlled by a microcontroller for a PV-tracking system with the ability to move on two axes. The spherical motors which have the linear and circular movement ability in three independent dimensions can be used for precisely tracking the sun as a solution. Roth et al. [8] has published a low-cost automatic closed-loop sun tracker, built and tested. A four-quadrant photodetector senses the position of the sun and two small DC motors move the instrument platform keeping the sun's image at the center of the four-quadrant photodetectors. The situation of tracking under cloudy conditions, when the sun is not visible, a computing program calculates the position of the sun and takes control of the movement, until the detector can sense the sun again. Rubio et al. [9] show a hybrid tracking system that consists of a combination of open-loop tracking strategies based on solar movement models and closed-loop strategies using a dynamic feedback controller. The results have been presented that show the benefits of the new strategy with respect to a classical open-loop strategy when errors in the estimation of the sun's position. Alata et al. [10] demonstrated the design and simulation of time controlled sun tracking system. The approach starts by generating the input/output data. Then, the subtractive clustering algorithm, along with least square estimation (LSE), generates the fuzzy rules that describe the relationship between the input/output data of solar angles that change with time. The fuzzy rules are tuned by an adaptive neurofuzzy inference system (ANFIS). This open-loop control system is designed for each of the previous types of sun tracking systems and the electrooptical trackers give very good results during good weather conditions. Some studies [11-13] also use four photoresistors with cylindrical shades, electrooptical unit, or electrooptical unit as a sun sensor. Its controller contains differential amplifiers, comparators, and output components to provide fairly good accuracy and reliable operation. Abdallah and Nijmeh [14] investigated the two axes tracking and the results indicate that the two axes tracking surface showed a better performance with an increase in the collected energy of up to $41.34 \%$ compared with the fixed surface. Neville [15] presented a theoretical comparative study between the energy available to a two axes tracker, an east-west tracker and a fixed surface. It was found that the energy available to the ideal tracker is higher by $5-10 \%$ and $50 \%$ higher than the east-west tracker and the fixed surface, respectively. Sungur [16] designed aboth axes sun tracking system with programmable logic control and with the function of solar azimuth angle and solar altitude angle to follow the sun's position. He found that the energy more than $42.6 \%$ in the two-axes sun-tracking system was obtained when compared to the fixed system. Barakat et al. [17] made a two-axes sun tracking system between closed-loop system and with complicated typical electronic control circuits. They found that the energy available to the two-axes tracker is higher by $20 \%$. Bakos [18] developed the two-axes tracking using a continuous operation on the solar energy collected. He set the position of the photoresistors and the results indicate that solar energy on the moving surface was significantly larger (up to $46.46 \%$ ) than the fixed surface. Khalifa and Al-Mutawalli [19] investigated the effect of using a two-axis sun tracking system on the thermal performance of compound parabolic concentrators (CPC). They indicated that the tracking CPC collector showed a better performance with an increase in the collected energy of up to $75 \%$ compared with an identical fixed collector. Mousazadeh et al. [20] established equations to compare the relation between tracking collector and fixed collector about energy gains. This amount of energy can be obtained from tracking collector more $57 \%$ than fixed collector.

However, for solar stirling electrical generator, the idea of concentrating solar energy to generate electricity has ingeniously made use of the concept in concentrator optics especially for designing a specific geometry of reflectors or lenses to focus sunlight onto a small receiving heating element. Many previous researches also have discussed that many existing concentrator systems will produce nonuniform focused illumination [21, 22] and it will decrease the energy collection of the sun. In this paper, a concept came from a realization that the need to concentrate the solar energy with the available Fresnel concentrating lens on the heating element of stirling engine to have higher efficiency in power generation. Therefore, we design the active two-axis solar tracker with Fresnel lens to collect the thermal power of solar efficiently and to offer a steady heat source for photo-thermal applications.

\section{Experiments}

2.1. The Structure of Two-Axis Heat-Collection Solar Tracker System. The proposed two axes heat collection solar tracker system as shown in Figure 1 consists of the following three parts:

(i) the heat-collection Fresnel lens module, 

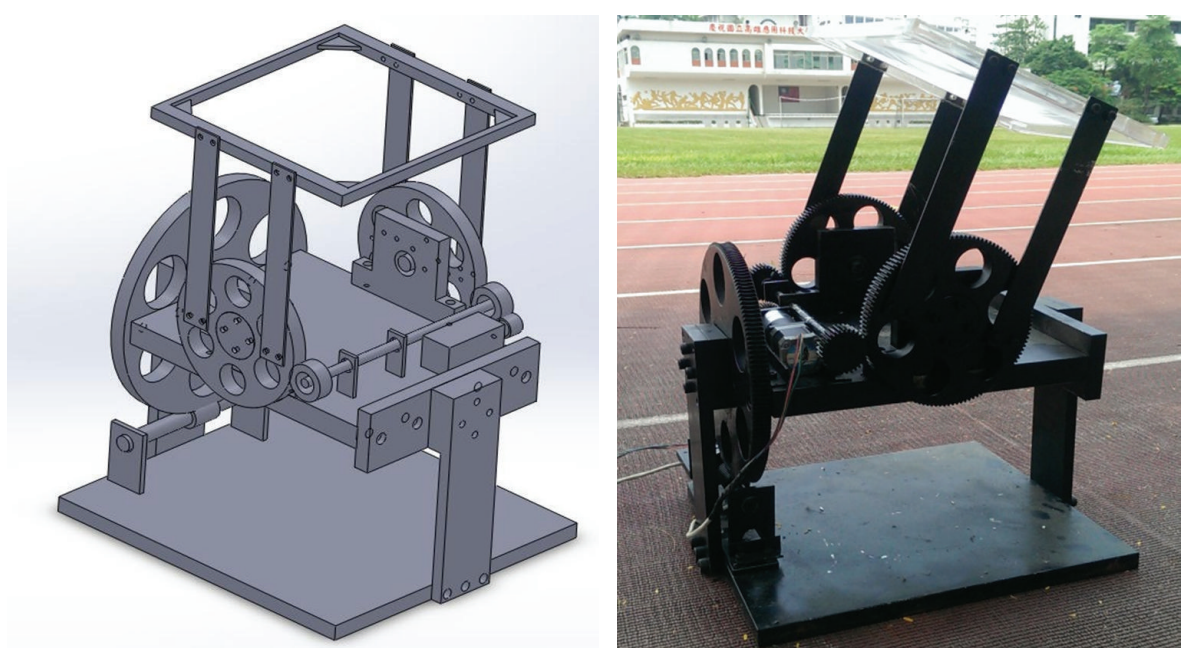

FIGURE 1: Schematic diagram of two-axis heat-collection solar tracker with constant focal distance.

(ii) the control system, and

(iii) the wireless data recorder system.

For the solar tracker system, increasing the solar input power and reducing the energy consumption of solar tracker are important goals in this paper. For reducing power consumption of solar tracker, we adopted the low-power motor to reduce energy loss and use the small gear to drive the large gear so the reducer will produced additional torque to move the polar axis structure of solar tracker. The heat collection Fresnel lens was used to concentrate the radiation power of sunlight and heat the heating head of stirling engine. However, because the position of heating head of stirling engine must put on the focus of sunlight, it is important to note that the focus of Fresnel lens must fix on the heating head to collect the solar heat energy at any time. For this purpose, two axis tracking method was used to trace the trajectory of the sun. Besides, because the change of sun tracks on north-south axis (N-S axis) is very small of a day, the stirling engine were arranged on the N-S axis plate to reduce the power assumption of moving step motor and the Fresnel lens was installed on the east-west axis (E-W axis) to collect the power of sun. This two-axis solar tracker was controlled by the step motor, reducer, worm, worm gear and make sure that the focus of Fresnel lens is always on the heating head of stirling engine. Besides, in our experiment, a portable wireless data acquisition system for temperature in real-time process dynamics was recorded by using the 802.15.4, ZigBee protocol, that is specially designed for the sensors network to facilitat data storage purposes. Zigbee is the only standardsbased wireless technology designed to address the unique needs of low-cost and low-power wireless sensor and control networks. This wireless data logger senses and monitors the variations in the temperature of simulated stirling head and the received temperature data can be displayed on a display and simultaneously on a computer.
2.2. Control System of Solar Tracker. The block diagram of our two-axis solar tracker as shown in Figure 2 composed of the following parts:

(i) the electromechanical movement mechanism,

(ii) the sensors unit, and

(iii) the control system software.

The mechanism of electromechanical displacement consists of two 12 volt-stepper motors, one for N-S axis and the other for E-W axis displacements and an electronic circuit. Light dependent resistor sensor (LDR sensor) is a component that has a resistance that changes with the light intensity that falls upon it. In order to track the position of the sun accurately, there are four light-dependent resistor sensors (LDR sensors, NSL19-M51 RS, an electrocomponents company, Northants) to identify the intensity of the sunlight. Each two light dependent resistor sensors were placed on the Fresnel lens along the east-west and north-south direction to control the N-S axis and E-W axis movement by two stepper motors, respectively. LDRs which produce a DC voltage level coincident with the light intensity of sun radiation detect the position of sunlight and then send the signals to the ADAM. So it is connected to one of the analog to digital converter (ADAM 4017/452) single pins to generate an 8-bits number (0-255) which tells the computer program some information about light intensity at that time and determine the device operating mode. The difference in illumination between each of the two sensors, the servo motion control card (SMCC) which produced pulse to driver, control the step motor, and do two axes operation (N-S and E-W axis motion), promotes an adjustment of the tracker position towards the sun. The angular interval spanned by the tracker goes from $80^{\circ}$ east up to $80^{\circ}$ west and $50^{\circ}$ south up to $50^{\circ}$ north. This operation mode forms a closed-loop system for sun tracking as illustrated in Figure 2. A proper program to control, monitor, and collect data was developed by using the software Labview to determine the solar angles. The control panel was set up at 

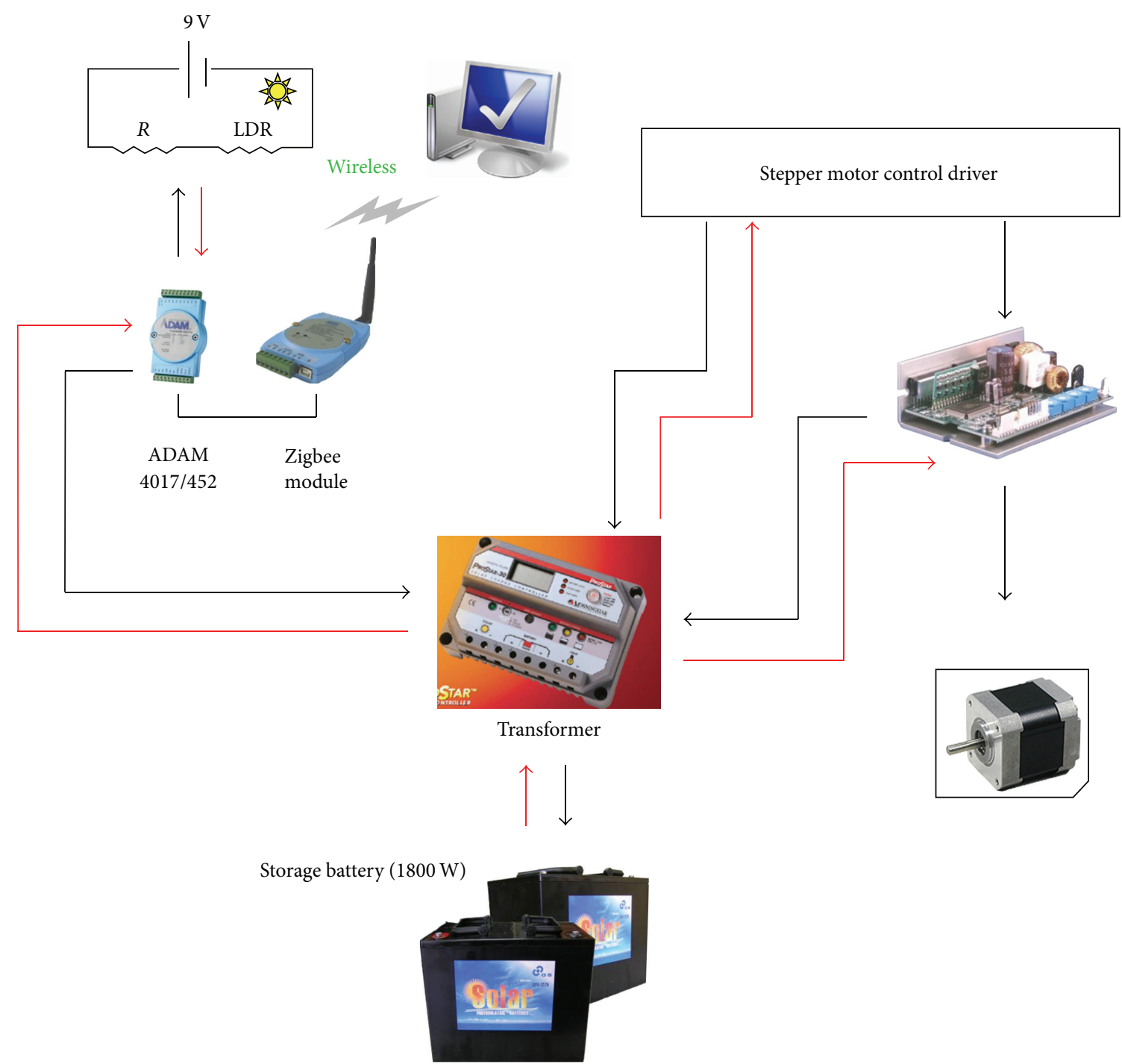

FIGURE 2: The schematic diagram of solar tracker control system.

the start time to receive and monitor the whole tracking process information about N-S axis or E-W axis directions. The relation between different sensor's voltage and SMCC control pulse can be defined from internal program to reduce the tracking times and the logic consequences of the controlling process of our heat collection solar racking system.

\section{Results and Discussion}

3.1. The Effect of the Structure of LDR Sensors. The design of the shadow mask of LDR sensors is an important factor because the better design will increase the accuracy of tracking sunlight and shorten the tracking time. In our experiment, the five different shadow types of LDR sensors: (a) 1/4 bowl type, (b) 1/2 bowl type, (c) bowl type, (d) board type, and (e) square type were designed to determine the best module for sun tracking as shown in Figure 3(a). The test between tracking pulses and angle was limited by the same rotating angles from $0^{\circ} \sim 60^{\circ}$ of solar tracker. In this experiment, we adjusted and fixed the fluorescent angle to measure the track of solar tracker as shown in Figure 3(b). If the tracking angles are the same, shorter tracking times means that the tracking speed is higher and the design of this shadow mask has better sensitivity. As the results form Figure 3(b), the board type is the best shadow mask design as the angle is less than 20 degrees, but $1 / 4$ bowl is the best shadow mask design to track the sunlight as the angle is greater than 20 degrees. According to these results, we found that the tracking time of sunlight is strongly dependent on the geometry of shadow mask. Therefore, in this paper, we use board type to be the shadow mask of LDRs because the angle of each tracking step is smaller than 20 degree.

3.2. The Measurement Method and Results of Heat Power of Sun. It is hard to measure the sun heat power because the power gain from the sun by Fresnel lens will cause very high temperature at focus. For this reason, in this paper, we designed the power gain measurement system by the temperature raising rate as illustrated in Figure 4. In our experiment, we put the copper block at the focus of Fresnel 


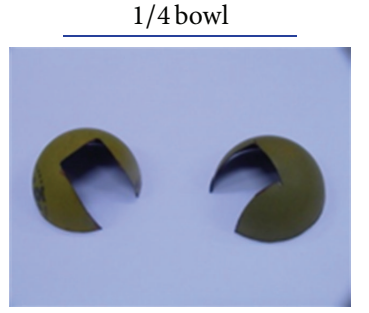

Bowl

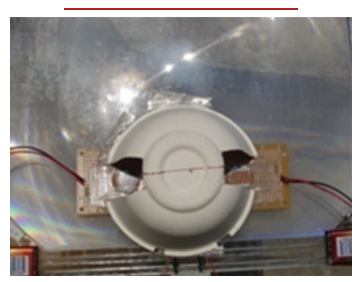

Square

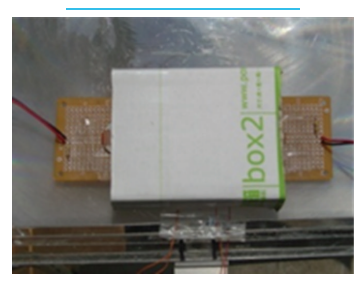

(a)
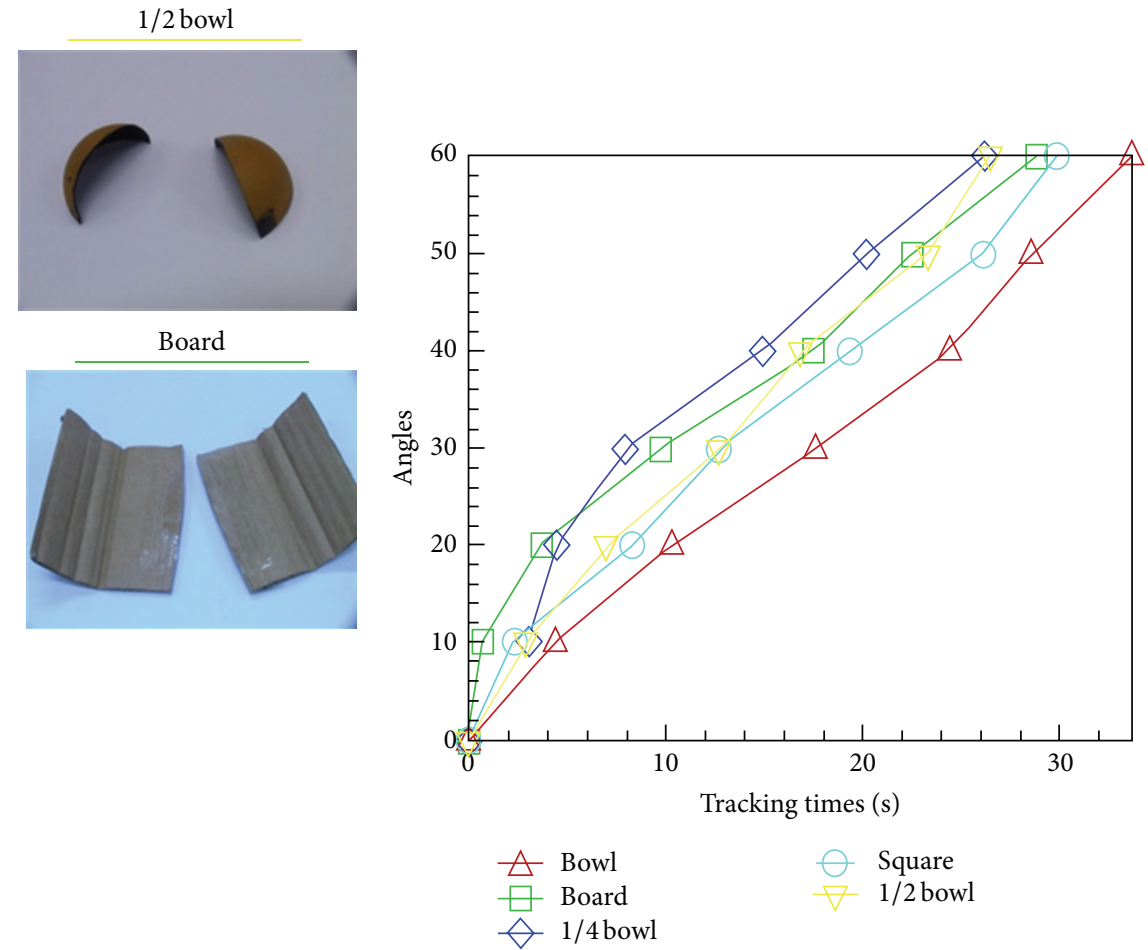

(b)

FIgURE 3: (a) The different shadow mask types of LDRs and (b) the tracking speed with different shadow masks.

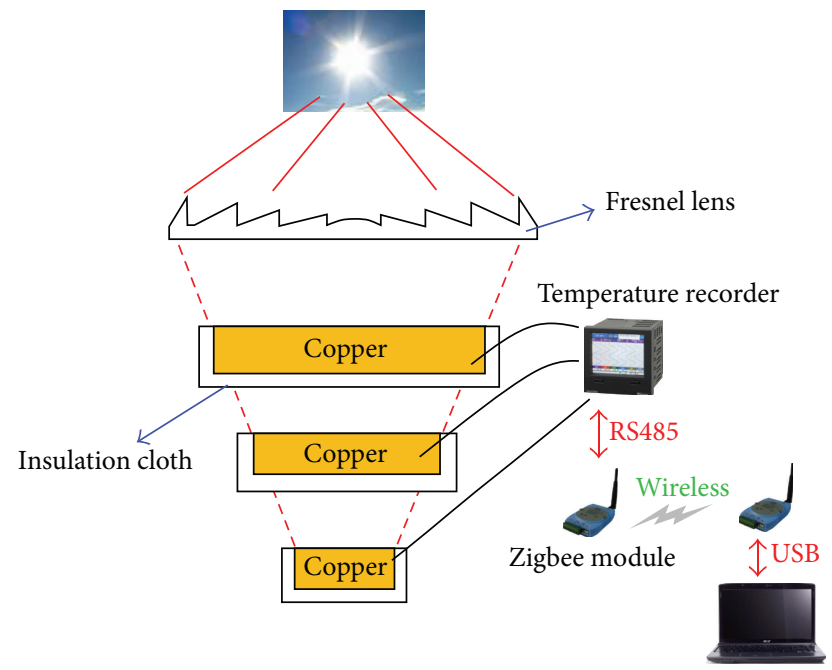

FIGURE 4: The schematic diagram of solar power measurement system.

lens on our two-axis solar tracker. There are three different area coppers, $19.63 \mathrm{~mm}^{2}, 1963.50 \mathrm{~mm}^{2}$, and $11309.73 \mathrm{~mm}^{2}$, which were used to record the temperature difference by the data recorder system and then used these temperature data to calculate the absorbing power. The asbestos insulation cloth was wrapped around the copper to reduce the heat loss. The power gain per unit area $W$ can be calculated from the following equation:

$$
W=\frac{\left(m \times s \times \Delta T /\left.\Delta t\right|_{\text {init }}\right)}{A},
$$

where $m$ is the mass of the copper, $s$ is the specific heat of the copper, $A$ is the area of the copper plate, $\Delta T$ is the range of temperature, and $\Delta t$ is the time (second) of this experiment. The $\Delta T /\left.\Delta t\right|_{\text {init }}$ is the slop at the initial period in Figure 5(a) and the slope difference between initial period and real period implies the heat loss of copper. As illustrated the schematic diagram in Figure 4, in order to make sure that the sunlight irradiate on the surface of copper totally to avoid the additional energy loss, the less focus length needs larger copper surface area. This method not only measures the power of sun irradiation but also finds the best heating area of stirling engine. As shown in Figure 5(a), the temperature slop at initial periodic means the smallest heat loss and the decrease of temperature slop at real periodic indicated that there are some heat loss such as heat transfer from copper and ambient or the energy storage by copper block. Therefore, the ideal power gain and heat loss of copper can be calculated form (1) at different focal length. The result indicated that at $345 \mathrm{~mm}$, focal length has best heating energy gain but large heat loss. This is because the larger temperature difference between copper and ambient air will cause the larger heat loss. Besides, Figure 5(b) shows the power of copper at different focal length and focal area at different times. The largest power of copper was acquired about $500 \mathrm{~W} / \mathrm{m}^{2}$ at 12:00 and the focal length was $345 \mathrm{~mm}$. The reason is that the large focal length has small focus range which means that the copper area is smallest. Small copper area which was heated by sunlight irradiation will reduce the heat loss from the copper and decrease the less storage energy of copper block. Therefore, if we want to get more heating power from 


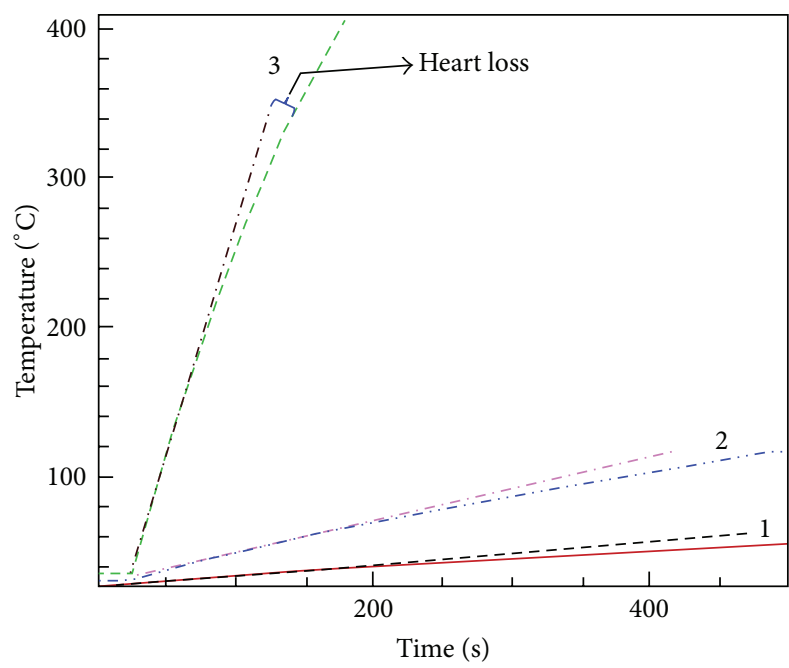

- (1) Focal length: $140 \mathrm{~mm}$

-... (2) Focal length: $240 \mathrm{~mm}$

- - - (3) Focal length: $345 \mathrm{~mm}$

(a)

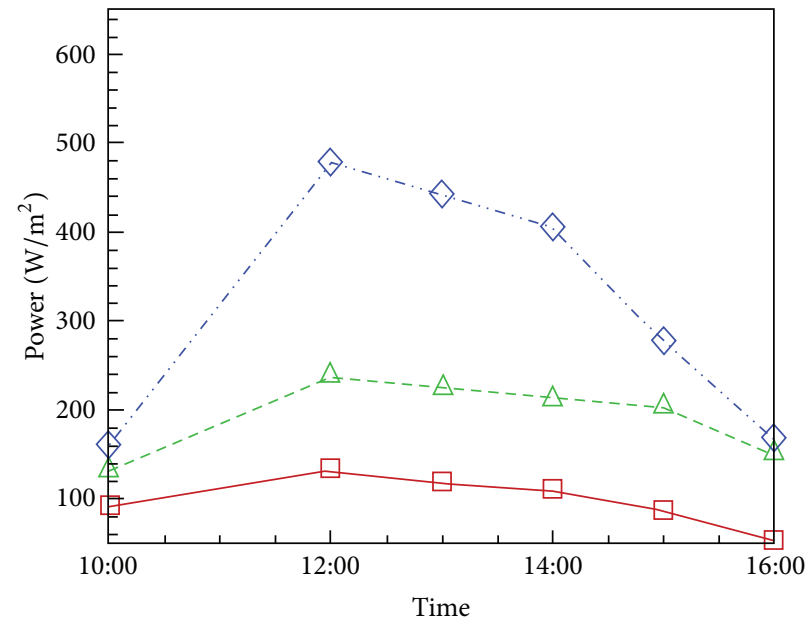

(1) Focal length: $345 \mathrm{~mm}$

-A- (2) Focal length: $240 \mathrm{~mm}$

(3) Focal length: $140 \mathrm{~mm}$

(b)

FIGURE 5: (a) The temperature raising rate and (b) the power gain of solar power measurement with different focal lengths.

sunlight, the heating head area of stirling engine should decrease as small as possible. Also shown in Figure 5(b), the solar heating power was larger during 12:00 to 13:00 than other times of the day at February 23 in Kaohsiung, Taiwan. It implied that if we want to get the sun irradiation power more efficiently, the time during 12:00-13:00 of the day is the best time period to get the sun irradiation power.

\subsection{Measurements of Two-Axis Tracking System under Normal} Weather Conditions. The solar power and the temperature of simulated stirling head experiments took place on 16 June 2012 from 09:00 to 16:00 in Kaohsiung, Taiwan, and the ambient temperature was $28^{\circ} \mathrm{C}$, the humidity $65 \%$, and the results are shown in Figure 6. During these experiments the weather conditions were very good and just a little cloudy in the sky at 12:25. The solar power was measured by our power gain measurement system as described earlier. As shown in Figure 6, the temperature response is slower and obtuse than the solar irradiation power. Because the insulation cloth was covered on the copper surface, the temperature is still maintained at a certain value even through the solar input energy decreases due to drifting clouds at 12:08. Moreover, from the data in Figure 6, the results also indicated that the best time to obtain the largest solar irradiation power is during 11:00-13:00.

\section{Conclusions}

In this paper an experimental study is performed to investigate the effect of closed-loop two-axis tracking on the solar heat energy collected for photo-thermal application. The hardware and software elements of the two-axes sun tracking system were designed and constructed. According to the results of the measurements performed in the present study,

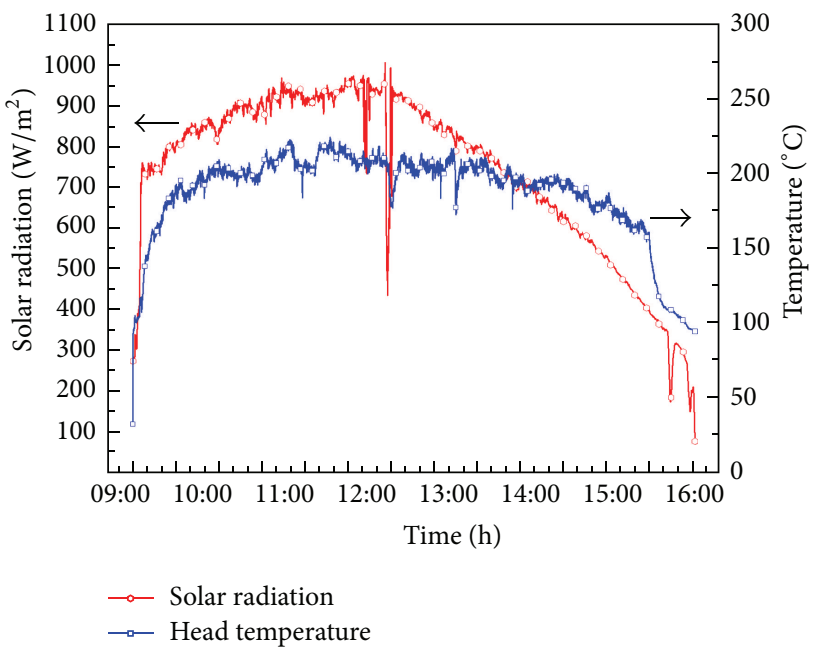

FIGURE 6: Time variation of solar irradiation power collection and heating head temperature by solar tracking system.

it can be concluded that proper shadow mask of LDR sensor will increase the accuracy and reduce the tracking time of tracking sun's radiation. Besides, in our experiment, we also design the heat power measurement system to measure the heat power of solar irradiation. Besides, the hourly values of solar radiation are added to obtain the total daily solar radiation and simulated stirling head temperature. The results indicated that the response of head temperature is slower than solar irradiation power without any heat loss, and if we want to get the solar heat power more efficiently, the time during 11:00-13:00 is the best time periods to get the sun's power. Moreover, in order to totally collect the solar 
thermal energy of the sunlight by Fresnel lens, letting the concentrative sunlight to totally illuminate on the heating element without any heat loss is very important.

\section{Acknowledgment}

The authors would like to thank the National Science Council of Taiwan, for partially supporting this research under Contract no. NSC 101-2221-E-151-010.

\section{References}

[1] J. A. Beltrán, J. L. González, S. Rubio, and C. D. GarcíaBeltrán, Fourth Congress of Electronics, Robotics and Automotive Mechanics, vol. 786, IEEE Computer Society Press, 2007.

[2] P. Roth, A. Georgiev, and H. Boudinov, "Cheap two axis sun following device," Energy Conversion and Management, vol. 46, no. 7-8, pp. 1179-1192, 2005.

[3] M. J. Clifford and D. Eastwood, "Design of a novel passive solar tracker," Solar Energy, vol. 77, no. 3, pp. 269-280, 2004.

[4] G. Mwithiga and S. N. Kigo, "Performance of a solar dryer with limited sun tracking capability," Journal of Food Engineering, vol. 74, no. 2, pp. 247-252, 2006.

[5] V. Poulek, "Testing the new solar tracker with shape memory alloy actors," in Proceedings of the 24th IEEE Photovoltaic Specialists Conference, pp. 1131-1133, December 1994.

[6] M. Abouzeid, "Use of a reluctance stepper motor for solar tracking based on a programmable logic array (PLA) controller," Renewable Energy, vol. 23, no. 3-4, pp. 551-560, 2001.

[7] Y. Oner, E. Cetin, H. K. Ozturk, and A. Yilanci, "Design of a new three-degree of freedom spherical motor for photovoltaictracking systems," Renewable Energy, vol. 34, no. 12, pp. 27512756, 2009.

[8] P. Roth, A. Georgiev, and H. Boudinov, "Design and construction of a system for sun-tracking," Renewable Energy, vol. 29, no. 3, pp. 393-402, 2004.

[9] F. R. Rubio, M. G. Ortega, F. Gordillo, and M. López-Martínez, "Application of new control strategy for sun tracking," Energy Conversion and Management, vol. 48, no. 7, pp. 2174-2184, 2007.

[10] M. Alata, M. A. Al-Nimr, and Y. Qaroush, "Developing a multipurpose sun tracking system using fuzzy control," Energy Conversion and Management, vol. 46, no. 7-8, pp. 1229-1245, 2005.

[11] S.-S. N. Rumala, "A shadow method for automatic tracking," Solar Energy, vol. 37, no. 3, pp. 245-247, 1986.

[12] W. A. Lynch and Z. M. Salameh, "Simple electro-optically controlled dual-axis sun tracker," Solar Energy, vol. 45, no. 2, pp. 65-69, 1990.

[13] V. Poulek and M. Libra, "New solar tracker," Solar Energy Materials and Solar Cells, vol. 51, no. 2, pp. 113-120, 1998.

[14] S. Abdallah and S. Nijmeh, "Two axes sun tracking system with PLC control," Energy Conversion and Management, vol. 45, no. 11-12, pp. 1931-1939, 2004.

[15] R. C. Neville, "Solar energy collector orientation and tracking mode," Solar Energy, vol. 20, no. 1, pp. 7-11, 1978.

[16] C. Sungur, "Multi-axes sun-tracking system with PLC control for photovoltaic panels in Turkey," Renewable Energy, vol. 34, no. 4, pp. 1119-1125, 2009.

[17] B. Barakat, H. Rahab, B. Mohmedi, and N. Naiit, "Design of a tracking system with photovoltaic panels," in Proceedings of the fourth Jordanian International Mechanical Engineering Conference (JIMEC '01), vol. 471, 2001.
[18] G. C. Bakos, "Design and construction of a two-axis Sun tracking system for parabolic trough collector (PTC) efficiency improvement," Renewable Energy, vol. 31, no. 15, pp. 2411-2421, 2006.

[19] A.-J. N. Khalifa and S. S. Al-Mutawalli, "Effect of two-axis sun tracking on the performance of compound parabolic concentrators," Energy Conversion and Management, vol. 39, no. 10, pp. 1073-1079, 1998.

[20] H. Mousazadeh, A. Keyhani, A. Javadi, H. Mobli, K. Abrinia, and A. Sharif, "A review of principle and sun-tracking methods for maximizing solar systems output," Renewable and Sustainable Energy Reviews, vol. 13, no. 8, pp. 1800-1818, 2009.

[21] H. Baiga, K. C. Heasmanb, and T. K. Mallick, "Non-uniform illumination in concentrating solar cells," Renewable and Sustainable Energy Reviews, vol. 16, no. 8, pp. 5890-5909, 2012.

[22] K. K. Chong, F. L. Siaw, C. W. Wong, and G. S. Wong, "Design and construction of non-imaging planar concentrator for concentrator photovoltaic system," Renewable Energy, vol. 34, no. 5, pp. 1364-1370, 2009. 

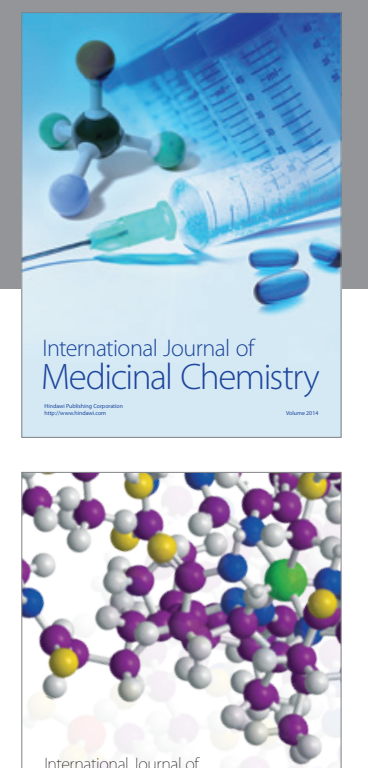

\section{Carbohydrate} Chemistry

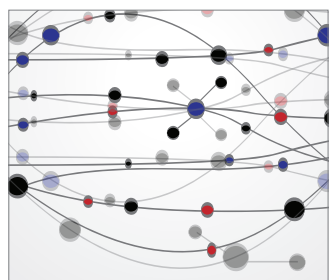

The Scientific World Journal
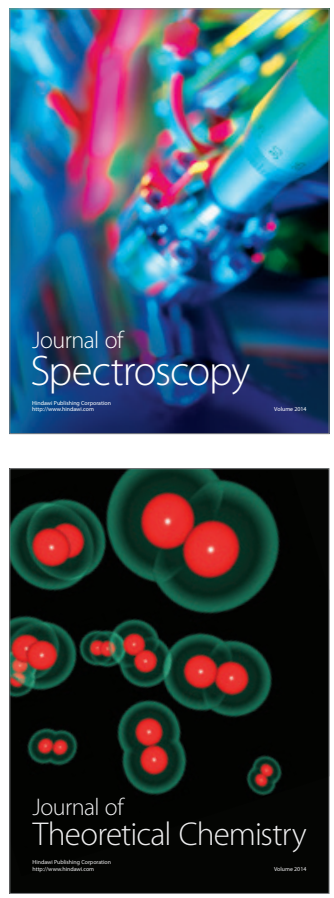
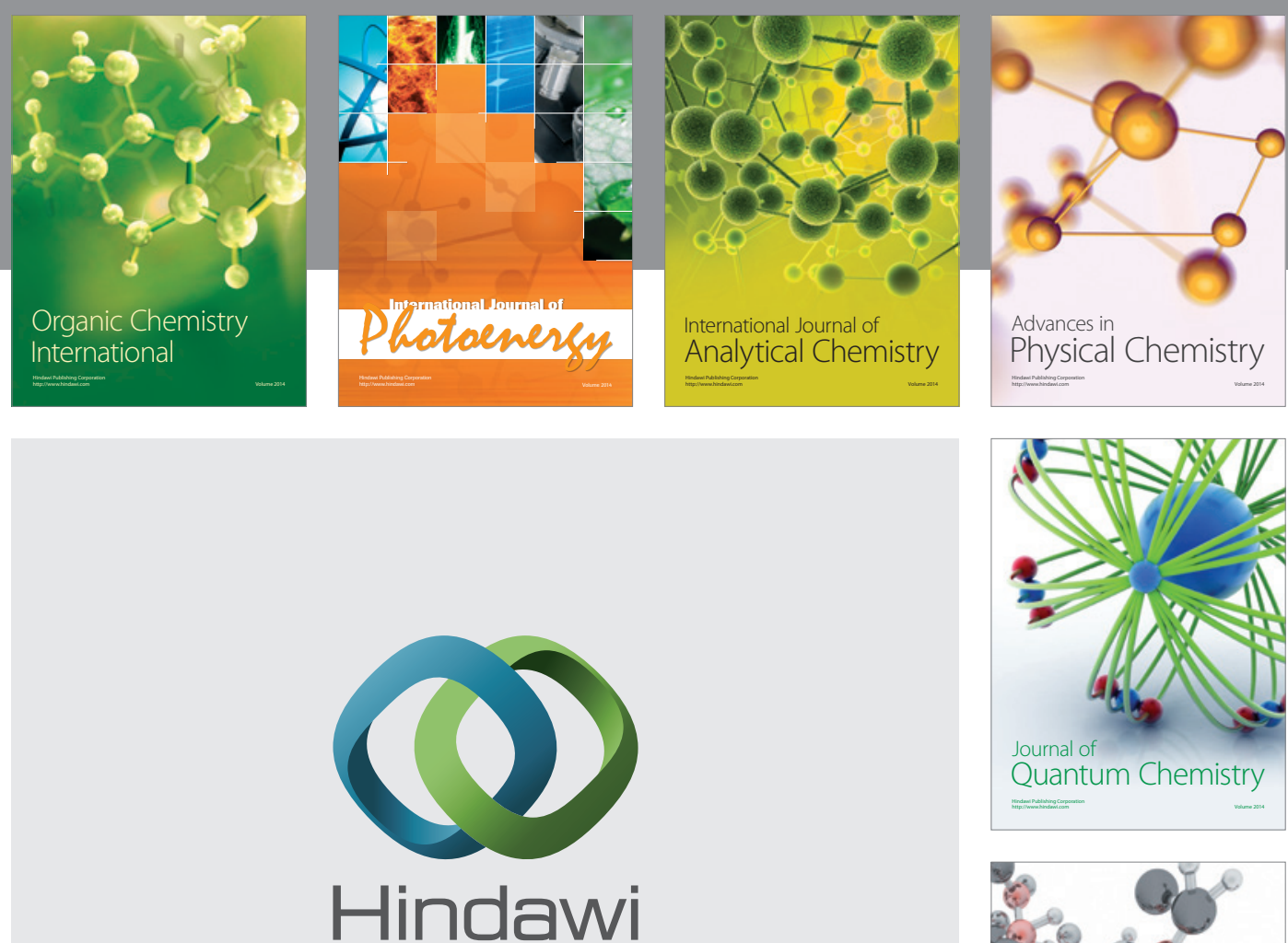

Submit your manuscripts at

http://www.hindawi.com

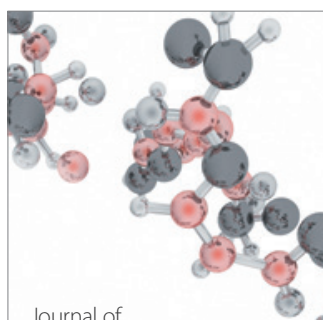

Analytical Methods

in Chemistry

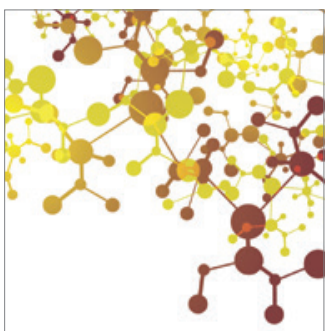

Journal of

Applied Chemistry

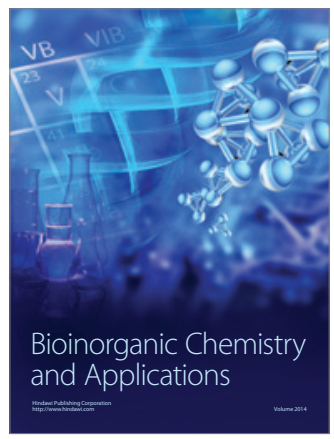

Inorganic Chemistry
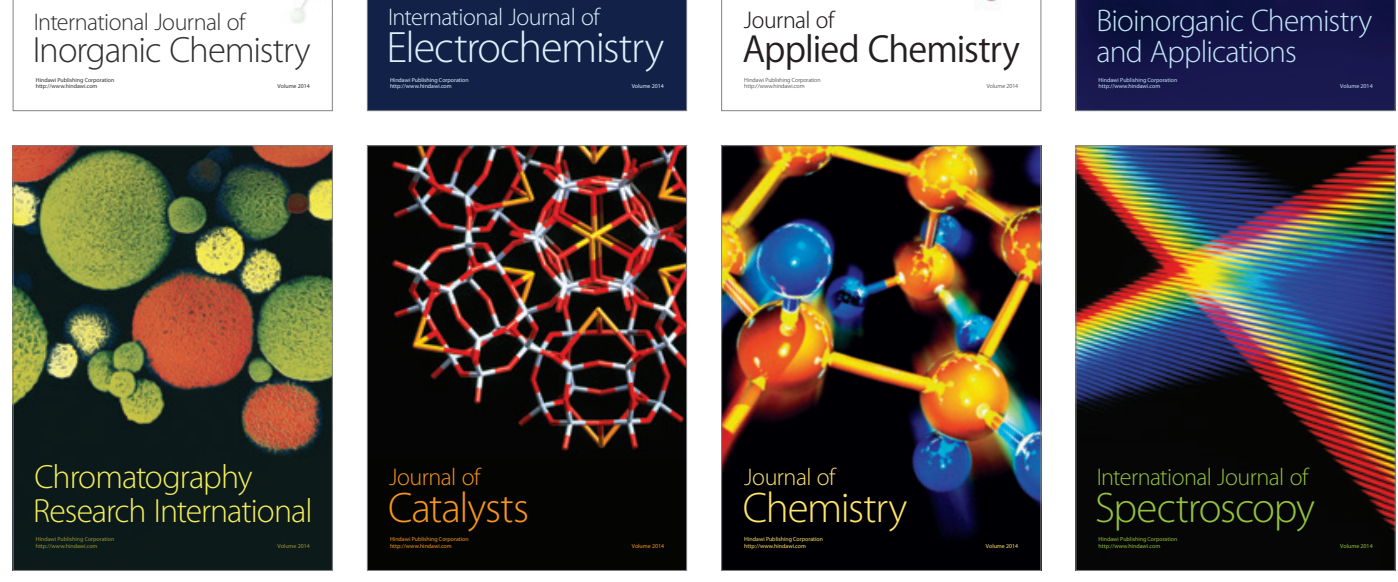